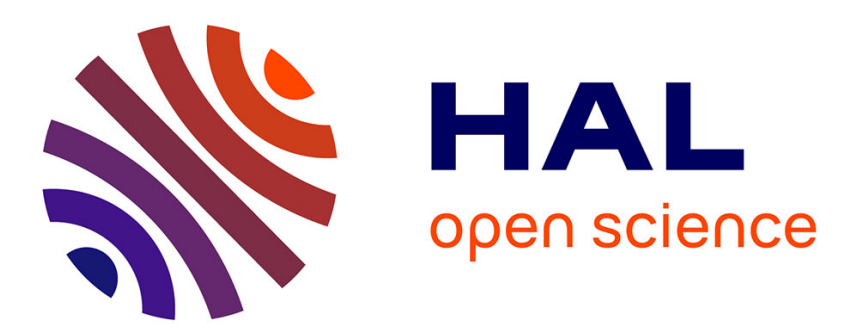

\title{
Extraction of Finite Element Basis Functions From the Cellular Topology of Meshes
}

Cédric Doucet, Isabelle Charpentier, Jean-Louis Coulomb, Christophe Guérin

\section{To cite this version:}

Cédric Doucet, Isabelle Charpentier, Jean-Louis Coulomb, Christophe Guérin. Extraction of Finite Element Basis Functions From the Cellular Topology of Meshes. IEEE Transactions on Magnetics, 2008, 44 (6), pp.726-729. 10.1109/TMAG.2007.915949 . hal-00287783

\section{HAL Id: hal-00287783 https://hal.science/hal-00287783}

Submitted on 24 Jun 2008

HAL is a multi-disciplinary open access archive for the deposit and dissemination of scientific research documents, whether they are published or not. The documents may come from teaching and research institutions in France or abroad, or from public or private research centers.
L'archive ouverte pluridisciplinaire $\mathbf{H A L}$, est destinée au dépôt et à la diffusion de documents scientifiques de niveau recherche, publiés ou non, émanant des établissements d'enseignement et de recherche français ou étrangers, des laboratoires publics ou privés. 


\title{
Extraction of Finite Element Basis Functions From the Cellular Topology of Meshes
}

\author{
Cédric Doucet $^{1}$, Isabelle Charpentier ${ }^{2}$, Jean-Louis Coulomb ${ }^{3}$, and Christophe Guérin ${ }^{4}$ \\ ${ }^{1}$ Laboratoire Jean Kuntzmann, UMR CNRS 5224, BP 53, Grenoble Cedex 9 38041, France \\ ${ }^{2}$ Laboratoire de Physique et Mécanique des Matériaux, UMR CNRS 7554, Ile du Saulcy, Metz Cedex 1 57045, France \\ ${ }^{3}$ Laboratoire G2ELab, UMR CNRS 5269, ENSIEG BP 46, Saint-Martin-d'Hères Cedex 38402, France \\ ${ }^{4}$ Cedrat SA, Meylan Cedex 38246, France
}

\begin{abstract}
This paper is devoted to the presentation of a simple but powerful topological approach for the computation of basis functions involved in the expression of finite element interpolants. It deals with the so-called nodal and edge elements but similar considerations can be made for the construction of facet ones. All standard cells are treated including pyramids.
\end{abstract}

Index Terms-Algebraic topology, finite element methods.

\section{INTRODUCTION}

$\mathbf{I}$ $\mathrm{N}$ THIS PAPER, a general and simple procedure for the construction of finite element basis functions corresponding to several families of mesh cells is developed. Some basis functions for tetrahedra, hexahedra, prisms and pyramids have already been proposed in [4], [5], [13] but they are given as formulas without any proof. The approach we propose here is constructive. It is based on a strict generalization of Whitney forms [12] on non-simplicial meshes. This extension has never been proposed before even though some heuristics are available in the literature [3], [7]. It leads to the formulation of new topological construction rules which simplify the computation of higher order basis functions. Indeed, higher order basis functions on quadrangles, hexahedra, prisms and pyramids are now only functions of simplicial ones which have mainly been studied by several authors [1], [11]. Moreover, these rules preserve hierarchy properties of simplicial families of finite elements.

\section{NODAL BASIS FunCtions}

Let us consider $\mathbb{R}^{n}$ as the ambient space. By definition, a $r$-cell $\sigma^{r}$ is a closed subset of $\mathbb{R}^{n}$ for which the smallest affine subspace $P\left(\sigma^{r}\right)$ containing it is of dimension $0 \leq r \leq n$. A 0 -cell is a point and a 1-cell is a line. In our case, 2-cells may be triangles (2-simplices) or parallelograms (2-parallelotopes) and 3 -cells may be tetrahedra (3-simplices), parallelepipeds (3-parallelotopes), prisms or pyramids.

\section{A. Join Cells}

As one knows, the simplicial nodal basis functions correspond to barycentric coordinates. Let $\sigma^{r}=p_{0}, \ldots, p_{r}$ denotes a $r$-simplex with vertices $p_{0}, \ldots, p_{r}$. We also define meas : $\sigma^{r} \rightarrow \mathbb{R}$ such that meas $\left(\sigma^{0}\right)=1$, meas $\left(\sigma^{1}\right)$ is the length of $\sigma^{1}$, meas $\left(\sigma^{2}\right)$ is the area of $\sigma^{2}$ and meas $\left(\sigma^{3}\right)$ is the volume of $\sigma^{3}$. Given a $r$-simplex $\sigma^{r}=p_{0} \ldots p_{r}$ and a 0 -simplex $p_{r+1} \notin$ $P\left(\sigma^{r}\right)$, we can construct a $(r+1)$-simplex $p_{0}, \ldots, p_{r} p_{r+1}$ by

Digital Object Identifier 10.1109/TMAG.2007.915949

Color versions of one or more of the figures in this paper are available online at http://ieeexplore.iee.org.

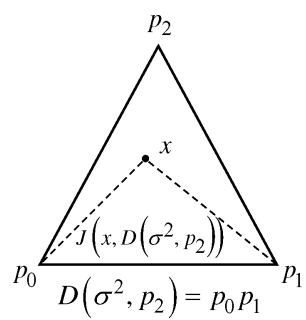

Fig. 1. A 2-simplex $\sigma^{2}=p_{0} p_{1} p_{2}$.

joining each vertex $p_{i}, i=0, \ldots, r$, to $p_{r+1}$ by an edge. This $(r+1)$-simplex will be denoted by $J\left(\sigma^{r}, p_{r+1}\right)$. If $p_{r+1} \in$ $P\left(\sigma^{r}\right)$, the join $J\left(\sigma^{r}, p_{r+1}\right)=0$ and it is said to be degenerated. Conversely, from a given simplex $\sigma^{r}=p_{0}, \ldots, p_{r}$, we can define a $(r-1)$-simplex by disjunction, i.e. by deleting a vertex from the simplex. The $(r-1)$-simplex $p_{0}, \ldots, p_{i-1} p_{i+1}, \ldots, p_{r}$ obtained by deleting $p_{i}$ from $\sigma^{r}$ will be denoted by $D\left(\sigma_{r}, p_{i}\right)$ (see Fig. 1). Using this notation, it is easy to verify that the corresponding barycentric coordinate $\mu_{p_{i}}^{r}(x)$ of a point $x \in \sigma^{r}$ associated to the vertex $p_{i}$ is given by

$$
\mu_{p_{i}}^{r}(x)=\frac{\operatorname{meas}\left(J\left(x, D\left(\sigma^{r}, p_{i}\right)\right)\right)}{\operatorname{meas}\left(\sigma^{r}\right)} \geq 0 .
$$

Then, $x$ is uniquely expressed under the form

$$
x=\sum_{i=0}^{r} \mu_{p_{i}}^{r}(x) p_{i} .
$$

We also recall that the barycentric coordinates form a partition of the unity, that is

$$
\forall x \in \sigma^{r}, \quad \sum_{i=0}^{r} \mu_{p_{i}}^{r}(x)=1 .
$$

The expression (2) is called an algebraic 0 -chain in combinatorial topology: it is a linear combination of 0 -cells (the vertices $\left.p_{i}\right)$. More generally, an algebraic $r$-chain is a linear combination of $r$-cells.

\section{B. Cartesian Cells}

We recall that the Cartesian product of two sets $X$ and $Y$ is the set

$$
X \times Y=\{(x, y), x \in X, y \in Y\} .
$$




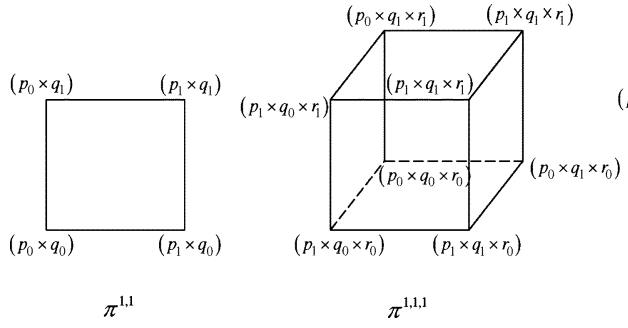

Fig. 2. Cartesian cells $\pi^{1,1}, \pi^{1,1,1}$ and $\pi^{2,1}$.

This topological construction naturally extends to the space of chains. Given a $r$-chain $\alpha=\sum_{i} a_{i} \sigma_{i}^{r}$ and a $s$-chain $\beta=$ $\sum_{j} b_{j} \sigma_{j}^{r}$, we can define the $(r+s)$-chain

$$
\alpha \times \beta=\sum_{i} \sum_{j} a_{i} b_{j}\left(\sigma_{i}^{r} \times \sigma_{j}^{s}\right)
$$

as the Cartesian product of $\alpha$ and $\beta$. From this concept, the extraction of nodal basis functions for parallelotopes and prisms is straightforward. Let $\sigma^{1}=p_{0} p_{1}, \tau^{1}=q_{0} q_{1}$ and $\rho^{1}=r_{0} r_{1}$ be three 1 -simplices and $\sigma^{2}=p_{0} p_{1} p_{2}$ be a 2 -simplex. Then, $\pi^{1,1}=\sigma^{1} \times \tau^{1}$ forms a 2-parallelotope, $\pi^{1,1,1}=\sigma^{1} \times \tau^{1} \times \rho^{1}$ forms a 3-parallelotope and $\pi^{2,1}=\sigma^{2} \times \rho^{1}$ forms a prism (see Fig. 2). Using relations (2) and (5), we deduce that any point $x \times y \in \pi^{1,1}$ is uniquely defined as

$$
\forall x \in \sigma^{1}, \forall y \in \tau^{1}, x \times y=\sum_{i, j=0}^{1} \mu_{p_{i} \times q_{j}}^{1,1}(x \times y)\left(p_{i} \times q_{j}\right)
$$

where $\mu_{p_{i} \times q_{j}}^{1,1}(x \times y)=\mu_{p_{i}}^{1}(x) \mu_{q_{j}}^{1}(y)$ are the nodal basis functions for $\pi^{1,1}$. More generally, one can observe that nodal basis functions on Cartesian products of points are products of nodal basis functions on these points. In particular, for any point $x \times$ $y \times z \in \pi^{1,1,1}$, nodal basis functions are $\mu_{p_{i} \times q_{j} \times r_{k}}^{1,1,1}(x \times y \times z)=$ $\mu_{p_{i} \times q_{j}}^{1,1}(x \times y) \mu_{r_{k}}^{1}(z)$ and for any point $x \times y \in \pi^{2,1}$, they are given by $\mu_{p_{i} \times q_{j}}^{2,1}(x \times y)=\mu_{p_{i}}^{2}(x) \mu_{q_{j}}^{1}(y)$. It can be shown that these nodal basis functions are a natural extension of simplicial Whitney 0-forms[12] on Cartesian cells.

\section{Cone Cells}

In topology the cone $C X$ over a set $X$ is the quotient space

$$
\begin{aligned}
C X & =(X \times I) /(X \times\{1\}) \\
& =\left\{(x, y) \in X \times I,\left(x_{1}, 1\right) \sim\left(x_{2}, 1\right), \forall x_{1}, x_{2} \in X\right\}
\end{aligned}
$$

where $I=[0,1]$ and $\sim$ denotes an equivalence relation on $X \times I$ induced by a surjective map $f: X \times I \rightarrow C X$ by saying that $x \sim y$ iff $f(x)=f(y)$ for all points $x, y \in X \times I$. We generalize this notion to cells as follows: given an $r$-cell $\sigma^{r}$ and a 1-simplex $\rho^{1}=r_{0} r_{1}$, we define the cone $C\left(\sigma^{r}, \rho^{1}\right)$ as the $(r+1)$-cell $C\left(\sigma^{r}, \rho^{1}\right)=\left(\sigma^{r} \times \rho^{1}\right) /\left(\sigma^{r} \times r_{1}\right)$ formed by $\sigma^{r}$ and $\rho^{1}$. The equivalence relation is then induced by a surjective map $\kappa_{a}: x \times y \in \sigma^{r} \times \tau^{1} \mapsto \pi_{1}(x) \times \pi_{2}(y)=\hat{x} \times \hat{y} \in C\left(\sigma^{r}, \tau^{1}\right)$ satisfying

$$
\begin{aligned}
\kappa_{a}\left(x \times r_{0}\right)= & \pi_{1}(x) \times \pi_{2}\left(r_{0}\right) \in C\left(\sigma^{r}, \rho^{1}\right), \\
& \forall x \in \sigma^{r}, \kappa_{a}\left(x \times r_{1}\right) \\
= & \hat{a} \times \pi_{2}\left(r_{1}\right) \in C\left(\sigma^{r}, \rho^{1}\right), \quad \forall x \in \sigma^{r}
\end{aligned}
$$
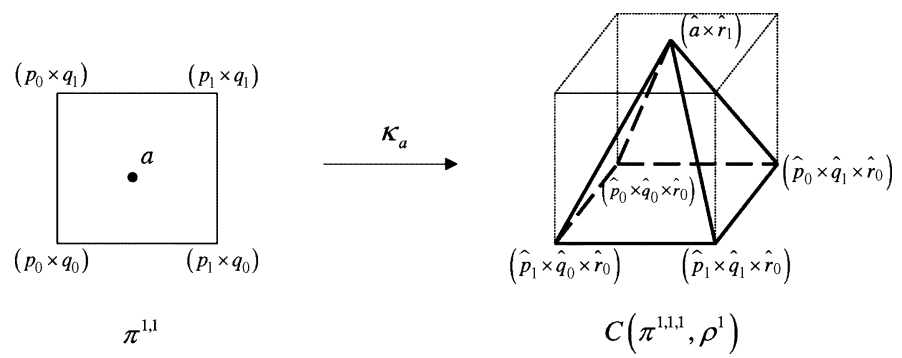

Fig. 3. Example of pyramid $C\left(\pi^{1,1}, \rho^{1}\right)$.

such that $\left(\pi_{1}\left(x_{1}\right) \times \pi_{2}\left(r_{1}\right)\right) \sim\left(\pi_{1}\left(x_{2}\right) \times \pi_{2}\left(r_{1}\right)\right)$ for all $x_{1}, x_{2} \in \sigma^{r}$ since $\kappa_{a}\left(x_{1} \times r_{1}\right)=\kappa_{a}\left(x_{2} \times r_{1}\right)$. The fixed vertex $\hat{a}$ is called the apex of the cone and can be chosen arbitrarily (see Fig. 3). Remembering that any point $x \times y \in \sigma^{r} \times \rho^{1}$ can be uniquely expressed under the form

$$
x \times y=\sum_{i} \sum_{j=0}^{1} \mu_{p_{i} \times r_{j}}(x \times y)\left(p_{i} \times r_{j}\right) .
$$

Considering the image $\kappa_{a}(x \times y)$, we obtain

$\hat{x} \times \hat{y}=\sum_{i} \mu_{p_{i} \times r_{0}}(x \times y)\left(\hat{p}_{i} \times \hat{r}_{0}\right)+\sum_{i} \mu_{p_{i} \times r_{1}}(x \times y)\left(\hat{a} \times \hat{r}_{1}\right)$.

As we observed previously, nodal basis functions on Cartesian products of points are products of respective nodal basis functions on these points because of (5). So

$$
\sum_{i} \mu_{p_{i} \times r_{1}}(x \times y)\left(\hat{a} \times \hat{r}_{1}\right)=\mu_{r_{1}}^{1}(y)\left(\hat{a} \times \hat{r}_{1}\right)
$$

since $\sum_{i} \mu_{p_{i}}(x)=1$ by definition. Using (11) and the fact that $x=\pi_{1}^{-1}(\hat{x})$ and $y=\pi_{2}^{-1}(\hat{y})$, we finally obtain

$$
\hat{x} \times \hat{y}=\sum_{i} \hat{\mu}_{\hat{p}_{i} \times \hat{r}_{0}}(\hat{x} \times \hat{y})\left(\hat{p}_{i} \times \hat{r}_{0}\right)+\hat{\mu}_{\hat{a}}(\hat{y})\left(\hat{a} \times \hat{q}_{1}\right)
$$

where $\hat{\mu}_{\hat{p}_{i} \times \hat{r}_{0}}(\hat{x} \times \hat{y})=\mu_{p_{i}}\left(\pi_{1}^{-1}(x)\right) \mu_{r_{0}}^{1}\left(\pi_{2}^{-1}(y)\right)$ and $\hat{\mu}_{\hat{a}}(\hat{y})=$ $\mu_{r_{1}}^{1}\left(\pi_{2}^{-1}(y)\right)$. These functions correspond to nodal basis functions associated with any type of cone. Nodal basis functions for pyramids are obtained by setting $\sigma^{r}=\pi^{1,1}$. A concrete example of such a cone is given in Section IV-A of this paper.

\section{EDGE BASIS FUNCTIONS}

The extraction of edge elements from the topology of cells lays on a procedure similar to the one we developed for nodal basis functions.

\section{A. The Simplicial Case}

Let $\sigma^{r}=p_{0} \ldots p_{r}$ be a $r$-simplex. We recall that the edge basis function on an edge $J\left(p_{i}, p_{j}\right), i \neq j$, corresponds to the basis function involved in the expression of the simplicial Whitney 1-form on this edge [12]. More precisely, this edge basis function is given by

$$
\mu_{J\left(p_{i}, p_{j}\right)}^{r}=\mu_{p_{i}}^{r} \nabla \mu_{p_{j}}^{r}-\mu_{p_{j}}^{r} \nabla \mu_{p_{i}}^{r}
$$

where the $\mu_{p_{i}}^{r}$ are given explicitly in (1). 


\section{B. The Case of Other Cells}

The computation of edge basis functions on other cells is really easy. Indeed, when cells are obtained from the Cartesian product of simplices, their vertices are Cartesian products of 0 -simplices by construction. Looking at their edges, we see that they are Cartesian products of 0 -simplices and joins. For example, the 2-parallelotope $\pi^{1,1}$ defined previously contains the four vertices $p_{i} \times q_{j}$, and the four edges $p_{i} \times J\left(q_{0}, q_{1}\right)$ and $J\left(p_{0}, p_{1}\right) \times q_{j}$ for $i, j=0,1$. The corresponding edge basis functions are simply the products of respective nodal basis functions and simplicial edge ones given in (13)

$$
\begin{aligned}
& \mu_{p_{i} \times J\left(q_{0}, q_{1}\right)}^{1,1}=\mu_{p_{i}}^{1} \mu_{J\left(q_{0}, q_{1}\right)}^{1}, i=0,1, \\
& \mu_{J\left(p_{0}, p_{1}\right) \times q_{j}}^{1,1}=\mu_{J\left(p_{0}, p_{1}\right)}^{1} \mu_{q_{j}}^{1}, j=0,1 .
\end{aligned}
$$

For the same reasons, edge basis functions on $\pi^{1,1,1}$ are $\mu_{p_{i} \times q_{j} \times J\left(r_{0}, r_{1}\right)}^{1,1,1}=\mu_{p_{i} \times q_{j}}^{1,1} \mu_{J\left(r_{0}, r_{1}\right)}^{1}, \mu_{p_{i} \times J\left(q_{0}, q_{1}\right) \times r_{k}}^{1,1,1}=$ $\mu_{p_{i} \times r_{k}}^{1,1} \mu_{J\left(q_{0}, q_{1}\right)}^{1}$ and $\mu_{J\left(p_{0}, p_{1}\right) \times q_{j} \times r_{k}}^{1,1,1}=\mu_{J\left(p_{0}, p_{1}\right)}^{1} \mu_{q_{j} \times r_{k}}^{1,1}$ for suitable indices $i, j, k$. Those of $\pi^{2,1}$ are $\mu_{p_{i} \times J\left(q_{0}, q_{1}\right)}^{2,1}=$ $\mu_{p_{i}}^{2} \mu_{J\left(q_{0}, q_{1}\right)}^{1}$ and $\mu_{J\left(p_{i}, p_{j}\right) \times q_{k}}^{2,1}=\mu_{J\left(p_{i}, p_{j}\right)}^{2} \mu_{q_{k}}^{1}$ for suitable indices $i, j, k$. Finally, the computation of edge basis functions for cone cells is also straightforward and we have

$$
\begin{aligned}
\hat{\mu}_{J\left(\hat{p}_{i}, \hat{p}_{j}\right) \times \hat{r}_{0}}^{r, 1}(\hat{x} \times \hat{y}) & =\hat{\mu}_{J\left(p_{i}, p_{j}\right) \times r_{0}}^{r, 1}\left(\pi_{1}^{-1}(x) \times \pi_{2}^{-1}(y)\right) \\
\hat{\mu}_{J\left(\hat{a} \times \hat{r}_{1}, \hat{p}_{i} \times \hat{q}_{0}\right)}^{r, 1} & =\hat{\mu}_{\hat{a} \times \hat{r}_{1}}^{r, 1} \nabla \hat{\mu}_{\hat{p}_{i} \times \hat{r}_{0}}^{r, 1}-\hat{\mu}_{\hat{p}_{i} \times \hat{r}_{0}}^{r, 1} \nabla \hat{\mu}_{\hat{a} \times \hat{r}_{1}}^{r, 1}
\end{aligned}
$$

from (13) and (14).

\section{Extraction of Finite Element Spaces}

Let $K \subset \mathbb{R}^{n}$ be a mesh cell, $P_{k}$ a functional space of finite $\operatorname{dimension} \operatorname{dim}\left(P_{k}\right)=m$. The dual space $P_{k}^{*}$ of $P_{k}$ is a $m$-dimensional space of linear forms $\phi_{i}: P \rightarrow \mathbb{R}$ called degrees of freedom such that any $p \in P_{K}$ can be uniquely written under the form

$$
p=\sum_{i=0}^{m} \phi_{i}(p) \mu_{i}
$$

We propose to identify suitable spaces $P_{K}$ for both nodal and edge finite elements from the results we obtained in the previous section.

\section{A. Lagrangian Spaces}

Let $x_{1}, \ldots, x_{n}$ denote the coordinates of a point $x$ in the canonical basis of $\mathbb{R}^{n}$ and $\alpha=\left(\alpha_{1}, \ldots, \alpha_{n}\right) \in \mathbb{N}_{+}^{n}$ where $\mathbb{N}_{+}$ denotes the set of positive integers. We set

$P_{i}^{k}=\operatorname{span}\left\{x_{i_{1}}^{\alpha_{1}} \ldots x_{i_{r}}^{\alpha_{r}}, \sum_{j=1}^{r} \alpha_{i_{j}} \leq k, i_{1}, \ldots, i_{r} \in I\right\}$.

Given two finite dimensional spaces $V=\operatorname{span}\left\{v_{1}, \ldots, v_{l}\right\}$ and $W=\operatorname{span}\left\{w_{1}, \ldots, w_{m}\right\}$, we define the operation

$$
V \otimes W=\operatorname{span}\left\{v_{i} w_{j}, 1 \leq i \leq l, 1 \leq j \leq m\right\}
$$

which can be interpreted as a tensor product. If $I_{n}$ denotes the set of the $n$ first strictly positive integers, we identify

$$
P_{I_{n}}^{1}=\operatorname{span}\left\{\mu_{p_{i}}^{r}(x), 0 \leq i \leq n\right\}
$$

as the functional space for $n$-simplicial nodal basis functions by expressing $x$ in the canonical basis of $\mathbb{R}^{n}$. From relation (6), we identify

$$
\bigotimes_{i=1}^{2} P_{\{i\}}^{1}=\operatorname{span}\left\{\mu_{p_{i} \times q_{j}}^{1,1}(x \times y), 0 \leq i, j \leq 1\right\}
$$

as the functional space corresponding to the cell $\pi^{1,1}$. Similarly, we find that functional spaces for cells $\pi^{1,1,1}$ and $\pi^{2,1}$ are the spaces $\bigotimes_{i=1}^{3} P_{\{i\}}^{1}$ and $\bigotimes_{i=1}^{2} P_{I_{i}}^{1}$ respectively. The case of the pyramid $C\left(\pi^{1,1}, \rho^{1}\right)$ is not generic since it depends on the choice of the apex. As illustration, we describe the case of the centered pyramidal cell $C\left([-1,1]^{2},[0,1]\right)$ with apex $(0,0,1)$ and cone mapping $\kappa_{a}:((x, y), z) \mapsto((\hat{x}, \hat{y}), \hat{z})=(((1-z) x,(1-z) y), z)$ and we obtain from (12) that the corresponding functional space is the space of rational functions

$$
\operatorname{span}\left\{1, \hat{x}, \hat{y}, \hat{z}, \frac{\hat{x} \hat{y}}{1-\hat{z}}\right\}
$$

since $\pi_{1}^{-1}((x, y))=\left(\hat{x} /(1-\hat{z}), \hat{y} /(1-\hat{z})\right.$ and $\pi_{2}^{-1}(z)=\hat{z}$.

\section{B. Nédélec's Spaces}

By computing explicitly edge basis functions for each cell, we identify Nédélec's spaces for simplicial and Cartesian cells [9], [10]. The new result concerns the case of pyramids. Setting $\left(P_{0}\right)^{3}=\operatorname{span}\{(1,0,0),(0,1,0),(0,0,1)\}$, we identify the space

$$
\begin{aligned}
\left(P_{0}\right)^{3} & \oplus \operatorname{span}\left\{\left(\hat{y}, 0, \frac{\hat{x} \hat{y}}{1-\hat{z}}\right),\left(0, \hat{x}, \frac{\hat{x} \hat{y}}{1-\hat{z}}\right)\right\} \\
& \oplus \operatorname{span}\{(\hat{z}, 0,-\hat{x}),(0, \hat{z},-\hat{y})\} \\
& \oplus \operatorname{span}\left\{\left(\frac{\hat{y} \hat{z}}{1-\hat{z}}, \frac{\hat{x} \hat{z}}{1-\hat{z}}, \frac{\hat{x} \hat{y} \hat{z}}{(1-\hat{z})^{2}}-\frac{\hat{x} \hat{y}}{1-\hat{z}}\right)\right\}
\end{aligned}
$$

as the suitable functional space for $C\left([-1,1]^{2},[0,1]\right)$. This is a natural extension of Nédélec spaces for this cell. Especially, its dual space is the classical set of degrees of freedom

$$
\phi_{e}(p)=\int_{e} p \cdot \tau_{e} d \gamma, \quad p \in(22)
$$

defined for each edge $e$ of the pyramid where $\tau_{e}$ denotes the unitary tangent vector along $e$.

\section{Higher ORder Finite Element Basis Functions}

\section{A. Higher Order Whitney Forms}

The computation of higher order finite element basis functions is easily performed using our approach. The best way for defining higher order Whitney 0 -forms is to introduce artificial 


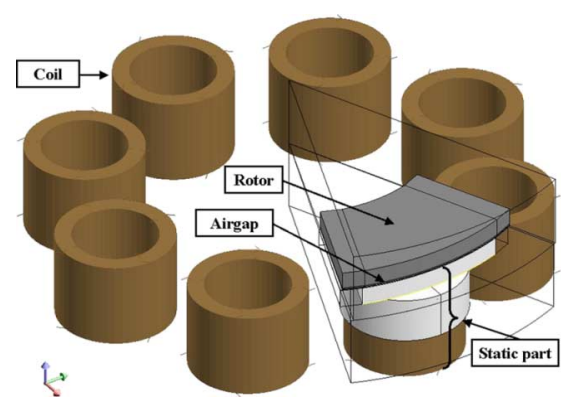

Fig. 4. Geometry of a brake retarder (only 1/8).

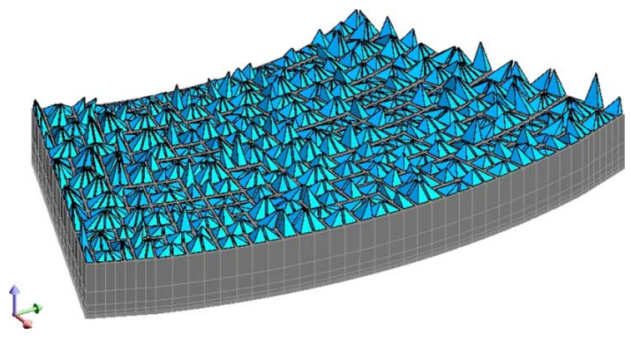

Fig. 5. Pyramidal elements on the surface of the rotor.

extra terms in (2). Let consider a $r$-simplex $\sigma^{r}=p_{0} \ldots p_{r}$. Remembering that $\sum_{j} \mu_{j}(x)=1$ and setting $p_{i j}=\left(p_{i}+p_{j}\right) / 2$, any point $x \in \sigma^{r}$ is uniquely expressed under the form $x=$ $\sum_{i, j} \mu_{i}(x) \mu_{j}(x) p_{i}$, that is

$$
x=\sum_{i} \mu_{i}(x)\left(2 \mu_{i}(x)-1\right) p_{i}+\sum_{i<j} 4 \mu_{i}(x) \mu_{j}(x) p_{i j} .
$$

The computation of higher order Whitney 0-forms for Cartesian cells only consists in reproducing the strategy previously used by computing respective Cartesian products of chains of the form (24). In the case of cone cells, the approach remains unchanged. The case of edge basis functions can be treated similarly: one knows that higher order edge basis functions on $r$-simplices are given by the formula $\mu_{J\left(p_{i}, p_{j}\right)}^{r,+}=\nabla\left(\mu_{i}^{r} \mu_{j}^{r}\right)$ and correspond to Nédélec's elements of the second kind[10]. Then, the corresponding edge basis functions are simply obtained by replacing terms of the form $\mu_{J(p, q)}^{r}$ by $\mu_{J(p, q)}^{r,+}$ in relations (14) and (15), etc.

\section{B. Remark on the Construction of Hierarchic Basis Functions}

Suppose now that a set of hierarchic basis functions for simplices are known. Then, since the Cartesian product of chains is distributive, this hierarchy will be preserved on Cartesian cells. This nice property is also true for cone cells since they are obtained from Cartesian cells by a change of variables.

\section{NUMERICAL VALIDATION}

We propose here to validate lowest-order nodal and basis functions on a $\mathrm{AC}$ steady state problem describing the brake retarder given in Fig. 4. Numerical results come from Flux software [6]. The rotor part is meshed by hexahedra and the static part by prisms. Coils are not meshed. The airbox containing the retarder (including the airgap) is discretized by means of tetrahedral elements. Some pyramidal elements are introduced to maintain conformity at the interface of the rotor and the air domain.

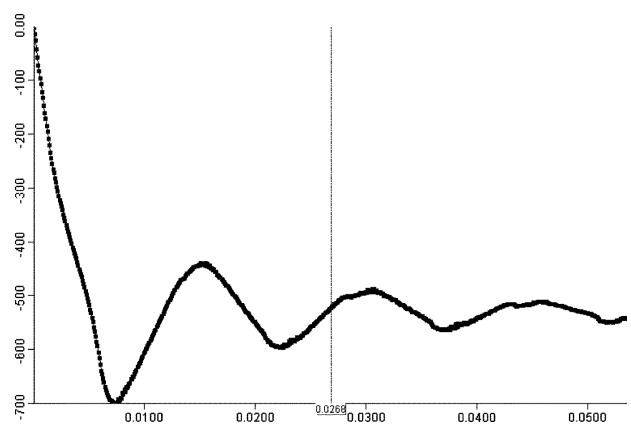

Fig. 6. Evaluation of the magnetic torque.

They are represented in Fig. 5. The equations of the problem are discretized using two versions of the $T 0-T-\phi$ formulation [8]. In the first case, the current vector potential $T$ and the magnetic potential $\phi$ are interpolated using nodal basis functions. The second version corresponds to the case where $T$ is interpolated using edge basis functions. The evolution of the magnetic torque is the same for the two versions and corresponds to the physical behavior of the brake (see Fig. 6).

\section{ACKNOWLEDGMENT}

The authors would like to thank Prof. A. Bossavit from Laboratoire de Génie électrique de Paris for his precious help in understanding underlying concepts involved in the construction of Whitney forms.

\section{REFERENCES}

[1] M. Ainsworth and J. Coyle, "Hierarchic finite element bases on unstructured tetrahedral meshes," Int. J. Numer. Meth. Eng., vol. 58, no. 14, pp. 2103-2130, 2003.

[2] A. Bossavit, "Generating Whitney forms of polynomial degree one and higher," IEEE Trans. Magn., vol. 38, no. 2, pt. 1, pp. 341-344, Mar. 2002.

[3] A. Bossavit, "Géométrie de l'électromagnétisme et éléments finis," in Électromagnétisme et Éléments Finis, G. Meunier, Ed. Paris: Hermès-Lavoisier, 2003, vol. 1, ch. 1, pp. 17-146.

[4] J.-L. Coulomb, F.-X. Zgainski, and Y. Maréchal, "A pyramidal element to link hexahedral, prismatic and tetrahedral edge finite elements," IEEE Trans. Magn., vol. 33, no. 2, pt. 2, pp. 1362-1365, Mar. 1997.

[5] P. Dular, J.-Y. Hody, A. Nicolet, A. Genon, and W. Legros, "Mixed finite elements associated with a collection of tetrahedra, hexahedra and prisms," IEEE Trans. Magn., vol. 30, no. 5, pt. 2, pp. 2980-2983, Sep. 1994.

[6] User's Guide. [Online]. Available: www.cedrat.com.

[7] V. Gradinaru and R. Hiptmair, "Whitney elements on pyramids," ETNA, vol. 8, pp. 154-168, 1999.

[8] G. Meunier, L. H. Tuan, and Y. Maréchal, "Computation of coupled problem of 3D Eddy current and electrical circuit by using $\mathrm{T}_{0}-\mathrm{T}-\phi$ formulation," IEEE Trans. Magn., vol. 34, no. 5, pt. 1, pp. 3074-3077, 1998.

[9] J.-C. Nédélec, "Mixed finite elements in $\mathbb{R}^{3}$," Numer. Math., vol. 35, pp. $315-341,1980$

[10] J.-C. Nédélec, "A new family of mixed finite elements in $\mathbb{R}^{3}$," Numer Math., vol. 50, pp. 57-81, 1986.

[11] J. Webb, "Hierarchal vector basis functions of arbitrary order for triangular and tetrahedral finite elements," IEEE Trans. Antennas Propagat., vol. 47, no. 8, pp. 1244-1253, Aug. 1999.

[12] H. Whitney, Geometric Integration Theory, 3rd ed. Harlow, U.K.: Addison-Wesley, 1999.

[13] F.-X. Zgainski, J.-L. Coulomb, Y. Maréchal, F. Claeyssen, and X. Brunotte, "A new family of finite elements: the pyramidal elements," IEEE Trans. Magn., vol. 32, no. 3, pt. 1, pp. 1393-1396, May 1996.

Manuscript received June 24, 2007. Corresponding author: J.-L. Coulomb (e-mail: Jean-Louis.Coulomb@g2elab.inpg.fr). 